\title{
SELECCIÓN DE LOS CRITERIOS QUE IDENTIFICAN LAS PRÁCTICAS Y CREENCIAS RELACIONADAS CON LA SALUD ORAL EN MUJERES GESTANTES *
}

\author{
${ }^{1}$ Liliana Alexandra Claro V., ${ }^{1}$ Adriana Sarmiento G., ${ }^{2}$ Claudia Milena Riveros A., ${ }^{3}$ Sonia Constanza Concha Sánchez. \\ ${ }^{1}$ Estudiante X Semestre F. de Odontología U. Santo Tomás. ${ }^{2}$ Odontóloga U. El Bosque, Especialista en Gerencia Hospitalaria ESAP, Especialista \\ en Relaciones Laborales UNAB, Especialista en Odontopediatría U. Santo Tomás, Docente U. Santo Tomás. ${ }^{3}$ Odontóloga \\ U. Santo Tomás, Especialista en Educación y Comunicación para la Salud UIS, Magíster en Epidemiología UIS, Docente U. Santo Tomás.
}

Autor responsable de correspondencia: Claudia Milena Riveros Alejo

Correo electrónico: milevale_odontopediatria@yahoo.es

\section{RESUMEN}

Objetivo: Seleccionar los criterios de las variables que identifican las prácticas y creencias relacionadas con salud oral en gestantes afiliadas a Salud Total EPS de Bucaramanga.

Materiales y métodos: La información recolectada en el presente estudio, se deriva de un ensayo clínico controlado, en el que la muestra la integraron 40 gestantes de las EPS, aleatoriamente asignadas al grupo experimental (20) y control (20). Las variables se retomaron de registros que incluían además de aspectos socio-demográficos: tipo de intervención, variables relacionadas con creencias y con prácticas, evaluadas antes y después de la intervención. El plan de análisis univariado implicó el cálculo de medidas de resumen según la naturaleza de las variables. Se aplicó análisis de factores con el propósito de priorizar los criterios que definían cada una de las variables relacionadas con prácticas y creencias en salud oral y se generaron las variables que consolidaban los aspectos de interés; con los criterios priorizados y variables generadas, se analizó su nivel de asociación según intervención aplicando test de Chi o exacto de Fisher para las cualitativas y, para las cuantitativas Prueba T de Student o test de rangos de Wilcoxon.

Resultados: Se lograron identificar los criterios que definían las variables relacionadas con creencias (boca sana, caries dental, sangrado de las encías, enseñanza de cepillado y creencias populares) y con prácticas (uso de elementos de higiene, frecuencia de cepillado, seda dental, actividades frente al sangrado de encías y ante el dolor dental). A partir de los criterios priorizados y de las variables generadas se lograron establecer diferencias estadísticamente significativas según intervención.

Conclusión: La priorización de variables permitió identificar aspectos básicos que podrían orientar los programas promocionales y preventivos dirigidos a las gestantes y evaluar el programa desarrollado, que parece ser eficaz en la modificación de las prácticas y creencias en las gestantes intervenidas. [Claro LA, Sarmiento A, Riveros CM, Concha SC. Identificación de prácticas y creencias relacionadas con la salud oral en mujeres gestantes. Ustasalud 2009; 8: 25 -36]

Palabras clave: Gestación, Prácticas y creencias, Atención odontológica.

\section{SELECTION OF THE CRITERIA THAT IDENTIFY PREGNANT KNOWLEDGE AND ATTITUDES ABOUT THEIR ORAL HEALTH}

\begin{abstract}
Purpose: To selected the criteria that identify the pregnant knowledge and attitudes about oral health in the women who attended Salud Total EPS in Bucaramanga.

Methods: The data collected (self administered questionnaire with closed answers) was taken from the pregnant women participant in a controlled clinical assay. Forty pregnant women were stratified in two groups (control and experimental). The variables evaluated were sociodemographic aspects, intervention type, knowledge and attitudes before and after the treatment. Means, modes, medians, standard deviation were determined for all data. Bivariate analyses were performed by chi square test, Student's $t$ test and the Wilcoson test. For all tests, $\mathrm{p} \leq 0.05$ was considered statistically significant.

Results: The criteria that defined the variables related to beliefs (healthy mouth, dental decay, gingival bleeding, brushing education and popular beliefs) and to practices (use of hygiene elements, brushing frequency of, soothes dental, activities against gingival bleeding and the dental pain) was identified. From the prioritized criteria and of the generated variables they were managed to establish statistically significant differences according to intervention.

Conclusions: The variables selection permitted to identify the basic aspects that could orient and evaluated the promotion and prevention programs directed to this population. The program done was effective changing the pregnant knowledge and attitudes toward oral health in the pregnant women.
\end{abstract}

Key words: Pregnancy, Knowledge and attitudes, Dental consultation.

Recibido para publicación: 9 de mayo de 2009. Aceptado para publicación: 10 de julio de 2009 .

* Grupo de Investigación CYDUSTA. 


\section{INTRODUCCIÓN}

La gestación es una etapa en la vida de la mujer en la cual, un óvulo fecundado se desarrolla hasta el nacimiento. Este periodo trae consigo muchos cambios en su vida y en la de su familia; implica una gran responsabilidad, nuevas ilusiones y también temores para la madre. Durante este periodo de tiempo se presentan eventos psicológicos, fisiológicos, sistémicos, hormonales y sociales totalmente nuevos. Además, hay cambios en las prácticas y creencias relacionadas con la salud oral.

El embarazo como condición sistémica, puede generar modificaciones en el medio oral. Tanto los dientes como los tejidos de sostén pueden verse afectados al presentarse condiciones propicias para un mayor acúmulo de placa bacteriana. $\mathrm{Mu}-$ chas gestantes creen que puede deberse a que el bebé extrae el calcio de sus dientes; no obstante, se ha demostrado que en realidad se presenta un recambio mineral con la saliva debido al aumento en la frecuencia de consumo de alimentos lo que repercute en una modificación en los niveles del pH oral. ${ }^{1}$

Existen diversos mitos y creencias como una respuesta a los problemas dentales y periodontales producidos durante el embarazo, estableciéndose una asociación equivocada entre éste, el incremento de caries dental y la pérdida de dientes. Expresiones tales como "en cada embarazo se pierde un diente", o "los tratamientos odontológicos causan daño al bebé" son usualmente manifestadas lo que demuestra la gran necesidad de educación en este grupo poblacional. ${ }^{2,3}$.

En Colombia, el Ministerio de Protección Social reconoce la importancia de la educación en salud oral, y estipula que todas las gestantes deben asistir a una consulta odontológica con el fin de recibir una valoración clínica, establecer factores de riesgo para la enfermedad periodontal y recibir asesoría en prácticas de higiene oral. ${ }^{4}$

Para la futura madre, la medicina tradicional no es ni la verdadera, ni la única que genera salud. Esto la conduce a escuchar y creer en diferentes recomendaciones. Ella se siente libre de escoger qué tipo de indicaciones seguir, porque piensa que para su embarazo existen diversas formas de cuidado y que todas son valiosas. ${ }^{5-7}$

Actualmente, algunas gestantes reemplazan el tratamiento odontológico por prácticas caseras, debido al miedo que la consulta les genera. ${ }^{6}$ Las creencias o mitos culturales, la actitud de la familia y la experiencia o inexperiencia de la embarazada desempeñan un papel fundamental y deter- minante en el manejo del estrés que le produce la consulta odontológica. ${ }^{7-13}$.

La caries dental y la enfermedad periodontal constituyen un problema de salud pública que depende, en gran parte, del comportamiento de los individuos y de la implementación de medidas preventivas. Sin embargo, con las actividades, campañas y políticas de salud, no se ha logrado conseguir el impacto social esperado en la población. ${ }^{7}$

Al considerar lo anterior, se desarrolló un programa preventivo orientado a promover la salud oral en un grupo de gestantes vinculadas a una Empresa Promotora de Salud (EPS) de la ciudad de Bucaramanga. Se indagaron las creencias y prácticas relacionadas con su salud oral y se intentó determinar los consolidados relacionados con las variables de interés aunque en dicha investigación no se clarificaron cuáles eran los criterios que definían estos aspectos. ${ }^{14}$ Por lo tanto, se consideró importante retomar la información recolectada y seleccionar los criterios de las variables que identificaron las prácticas y las creencias relacionadas con la salud oral de la gestante.

\section{MATERIALES Y MÉTODOS}

La información recolectada en el presente estudio, se deriva de un ensayo clínico controlado cuya muestra estuvo integrada por 40 gestantes de las cuales 20 conformaron el grupo control y las 20 restantes, el grupo experimental. Se comparó el nivel de conocimiento antes y después de la intervención. Con el fin de determinar el universo, se indagó en la EPS el número de mujeres embarazadas vinculadas mensualmente al programa de control prenatal. Se obtuvieron los datos de agosto (48), septiembre (55) y octubre (56) de 2005, para un promedio de 53 gestantes. Sin embargo, de acuerdo con las estadísticas, aproximadamente el $20 \%$ (10 mujeres) podrían presentar abortos espontáneos, por lo cual se reclutaron 40 gestantes.

Se consideraron como criterios de inclusión ser gestante entre los 15 y 45 años de edad, en segundo y tercer trimestre de embarazo, adherida al programa de control prenatal, sistémicamente sana, haber firmado el consentimiento informado y estar afiliada a la EPS que aceptó participar en el estudio, en la ciudad de Bucaramanga.

Los criterios de exclusión fueron: ser paciente bajo tratamiento antibiótico, fumadora, con infecciones en el tracto genitourinario y quien planeara cambiar de residencia en los seis meses siguientes al primer interrogatorio. 


\section{Las variables recolectadas fueron:}

Variables sociodemográficas: edad, lugar de nacimiento, procedencia, estado civil, estrato socioeconómico, escolaridad, ocupación, embarazo deseado, madres primerizas.

Intervención: definida como la implementación o no del programa preventivo desarrollado en la investigación primaria.

Las relacionadas con creencias y prácticas que incluían las siguientes variables con los respectivos criterios que las analizaban y que fueron adaptados del cuestionario de representaciones y prácticas en salud oral aplicado en el ENSAB III: ${ }^{15}$

\section{Asociadas a creencias:}

Tener una boca sana es: los criterios incluidos para esta variable fueron tener los dientes blancos y parejos, no tener dolor, no tener caries dental "picas", tener los dientes completos, no tener mal aliento, no tener placa bacteriana o cálculos, no tener sangrado en las encías.

Los dientes se "pican" por: mal cepillado o no cepillado, consumo de dulces, descalcificación ósea, mala alimentación.

El sangrado o inflamación de las encías se produce por: mal cepillado o no cepillado, cepillado fuerte, problemas en el organismo, mala alimentación.

¿Quién le enseño a cuidarse la boca? Padres, maestros, odontólogos, radio/televisión, sola.

Está de acuerdo o no con las siguientes afirmaciones: En cada embarazo se pierde un diente; el uso de seda dental evita el sangrado de las encías; la crema dental es necesaria para la limpieza de la boca; tener los dientes amarillos significa que están dañados, si los padres tienen mala dentadura, los hijos también; sólo cuando hay dolor se visita al odontólogo; cuanta más crema se use, más limpios quedan los dientes; es mejor tener caja que tener los dientes naturales.

Es importante anotar que Creencias Consolidado se creó a partir de la aplicación del análisis de los factores con base en las variables priorizadas.

\section{Asociadas a prácticas:}

Elementos utilizados para limpiar sus dientes: cepillo, seda dental, crema dental, enjuague bucal, palillos, bicarbonato, sal, hierbas.

¿Cuándo cepilla los dientes? Al levantarse, después del desayuno, después del almuerzo, después de la comida (cena), antes de acostarse, nú- mero de veces al día en que se cepilla.

Si usa seda dental ¿Cuándo la emplea? Cuando le quedan restos de comida entre los dientes, cuando se acuerda, siempre que se cepilla para completar la higiene. Si no la usa: ¿Cuál es la razón? Le sangran o le duelen las encías, es costosa, no sabe usarla, falta de costumbre, no recuerda emplearla, no la conoce.

¿Qué hace cuando le sangran las encías? Consulta al odontólogo, cambia la forma de cepillarse, hace enjuagues con agua, usa la seda dental, utiliza medicamentos, usa remedios caseros, se cepillas más veces, no hace nada.

¿Qué hace cuando le duele una muela? Consulta al odontólogo, toma antibióticos, toma analgésicos, usa remedios caseros.

Prácticas Consolidado también se creó a partir de la aplicación del análisis de los factores con base en las variables priorizadas.

Para realizar el procedimiento se retomaron los cuestionarios que previamente habían sido diligenciados por las gestantes ${ }^{14} \mathrm{y}$ se analizaron los datos. Para verificar los procesos de sistematización de la información y del procesamiento se realizó una prueba con nueve formatos; a partir de ella se efectuaron modificaciones en la base de datos y correctivos en el procesamiento estadístico.

Posteriormente, se procedió a rectificar el proceso de codificación de la información y se sistematizó por duplicado en el programa Excel; ${ }^{16}$ la información obtenida se exportó al programa EPIINFO 6.04 y mediante la opción Validate se verificó la calidad de la digitación, se efectuaron la correcciones pertinentes y, finalmente, se exportó al paquete estadístico Stata 8.0 para su adecuado procesamiento. ${ }^{17,18}$

Para el análisis univariado, se describieron las características socio-demográficas; ${ }^{19}$ para ello se calcularon promedios para las variables cuantitativas y proporciones para las variables cualitativas. Posteriormente, las variables creencias y prácticas, y los criterios que las definen se evaluaron a través de un análisis de factores con el fin de reducir el número de criterios que se analizaron por cada grupo de variables y de redefinirlas. ${ }^{20}$ Se partió con la elaboración de las matrices de correlación, se realizaron análisis de componentes principales y se obtuvo el peso de cada criterio, se priorizaron y se generaron variables para finalmente obtener variables que definían creencias y prácticas en las gestantes. 
Con las variables priorizadas se realizó el análisis bivariado que consistió en el cruce de las variables priorizadas sobre prácticas y creencias en salud oral recolectadas antes y después de la intervención, según la implementación o no del programa preventivo; para ello se aplicó la prueba de $\mathrm{Chi}^{2}$ o exacto de Fisher para variables cualitativas y para las cuantitativas Prueba t de Student o test de rangos de Wilcoxon, según la distribución de los datos. ${ }^{19}$ Todo el análisis consideró un nivel de significancia $\alpha=0.05$.

Los aspectos éticos acatados en esta investigación estuvieron regidos por la Resolución 008430 del 4 de Octubre de 1993 al considerar siempre los principios relacionados con el respeto a la dignidad, la protección de los derechos, el bienestar, la seguridad y la privacidad de los sujetos. ${ }^{21}$

\section{RESULTADOS}

\section{Análisis univariado}

En total se estudiaron 40 maternas afiliadas a Salud Total EPS en la ciudad de Bucaramanga, con una edad promedio de $25.33 \pm 5.6$ años; el 55\% (22) había nacido en Bucaramanga y el $92.5 \%$ (37) procedía de la zona urbana.

La mayor frecuencia de estado civil fue el de casada en un $42.5 \%$ (17), seguido de un $40 \%$ (16) que vivía en unión libre. El 47.5\% (19) era de una estrato socioeconómico medio-bajo y el $75 \%$ (30) de las gestantes había terminado su bachillerato. El $52.5 \%$ (21) trabajaba fuera del hogar. Sobresale un $50 \%$ (20) de embarazos planeados y una mediana gestacional de $25.7 \pm 3.9$ semanas. El $72.5 \%$ (29) de las mujeres eran madres primerizas (Tabla1). ${ }^{14}$

Tabla 1. Descripción de variables sociodemográficas y las relacionadas con el embarazo en las gestantes afiliadas a una EPS en Bucaramanga analizadas de forma global y según intervención. ${ }^{14}$

\begin{tabular}{|c|c|c|c|c|}
\hline VARIABLE & $\begin{array}{c}\text { Global } \\
n=40 \\
\text { Frec. }(\%)\end{array}$ & $\begin{array}{c}\text { Intervenidas } \\
\mathbf{n}=\mathbf{2 0} \\
\text { Frec. }(\%)\end{array}$ & $\begin{array}{c}\text { No intervenidas } \\
\mathbf{n}=\mathbf{2 0} \\
\text { Frec. }(\%)\end{array}$ & $\mathbf{P}^{*}$ \\
\hline Población & $40(100)$ & $20(50)$ & $20(50)$ & -- \\
\hline $\begin{array}{l}\text { Edad } \\
15 \text { a } 20 \\
26 \text { a } 30 \\
\text { Más de } 30\end{array}$ & $\begin{array}{l}24(60) \\
9(22.5) \\
7(17.5)\end{array}$ & $\begin{array}{l}11(55) \\
5(25) \\
4(20)\end{array}$ & $\begin{array}{l}13(65) \\
4(20) \\
3(15)\end{array}$ & 0.827 \\
\hline $\begin{array}{l}\text { Lugar de nacimiento } \\
\text { Otro } \\
\text { Bucaramanga }\end{array}$ & $\begin{array}{l}18(45) \\
22(55)\end{array}$ & $\begin{array}{l}10(55.56) \\
10(45.45)\end{array}$ & $\begin{array}{c}8(44.44) \\
12(54.55)\end{array}$ & $0.525^{* *}$ \\
\hline $\begin{array}{l}\text { Procedencia } \\
\text { Zona rural } \\
\text { Zona urbana }\end{array}$ & $\begin{array}{c}3(7.5 \%) \\
37(92.5 \%)\end{array}$ & $\begin{array}{c}1(33.33) \\
19(51.35)\end{array}$ & $\begin{array}{c}2(66.67) \\
18(48.65)\end{array}$ & $1^{*}$ \\
\hline $\begin{array}{l}\text { Estado civil } \\
\text { Casada } \\
\text { Soltera } \\
\text { Unión libre } \\
\text { Viuda }\end{array}$ & $\begin{array}{l}17(42.5) \\
6(15) \\
16(40) \\
1(2.5)\end{array}$ & $\begin{array}{c}10(58.82) \\
2(33.33) \\
7(43.75) \\
1(100)\end{array}$ & $\begin{array}{l}7(41.18) \\
4(66.67) \\
956.25) \\
---\end{array}$ & $0.520^{*}$ \\
\hline $\begin{array}{l}\text { Estrato socio económico } \\
1 \text { bajo-bajo } \\
2 \text { bajo } \\
3 \text { medio-bajo } \\
4 \text { medio } \\
5 \text { medio alto }\end{array}$ & $\begin{array}{c}1(2.5) \\
15(37.5) \\
19(47.5) \\
4(10) \\
1(2.5)\end{array}$ & $\begin{array}{c}-- \\
8(53.33) \\
8(42.11) \\
3(75) \\
1(100)\end{array}$ & $\begin{array}{c}1(100) \\
7(46.67) \\
11(57.89) \\
1(25) \\
---\end{array}$ & $0.472^{* *}$ \\
\hline $\begin{array}{l}\text { Escolaridad } \\
\text { Primaria } \\
\text { Secundaria } \\
\text { Pregrado } \\
\text { Postgrado }\end{array}$ & $\begin{array}{l}3(7.5) \\
30(75) \\
6(15) \\
1(2.5)\end{array}$ & $\begin{array}{c}1(33.33) \\
17(56.67) \\
2(33.33) \\
---\end{array}$ & $\begin{array}{c}2(66.67) \\
13(43.33) \\
4(66.67) \\
1(100)\end{array}$ & $0.469^{* *}$ \\
\hline $\begin{array}{l}\text { Ocupación } \\
\text { Trabaja fuera de casa } \\
\text { Hogar }\end{array}$ & $\begin{array}{l}21(52.5) \\
19(47.5)\end{array}$ & $\begin{array}{c}8(38.10) \\
12(63.16)\end{array}$ & $\begin{array}{c}13(61.90) \\
7(36.84)\end{array}$ & $0.113^{* *}$ \\
\hline Embarazo deseado & $20(50)$ & $9(45)$ & $11(55)$ & $0.527^{* *}$ \\
\hline Madres primerizas & $29(72.5)$ & $13(45)$ & $16(55.2)$ & $0.288^{* *}$ \\
\hline
\end{tabular}

Exacto de Fisher * $\mathrm{Chi}^{2}{ }^{* *}$ 


\section{Análisis de factores}

Para cada una de las variables se elaboró la matriz de correlación, el respectivo análisis de factores, las pendientes de Catell correspondientes y, a partir de ello, se efectúo el proceso de priorización de los criterios que mejor representaban cada una de las variables, y/o se generó la nueva variable que definiría la variable analizada. Los criterios priorizados y los respectivos coeficientes de puntajes para creencias y prácticas se evidencian en las tablas 2 y 3.

Es importante destacar que para la variable sangrado e inflamación de encías (en grupo de creencias) y consolidado de creencias populares fue posible generar una nueva variable que agrupaba a todos los criterios que los integraban y por ello no se reportan los criterios que la representan ni el coeficiente de puntaje.

Tabla 2. Variables y sus criterios priorizados mediante análisis de factores para los conocimientos sobre creencias de las gestantes en salud oral.

\begin{tabular}{llc}
\hline \multicolumn{1}{c}{ VARIABLE } & \multicolumn{1}{c}{ Criterios Priorizados } & Coeficientes de Puntajes \\
\hline \multirow{2}{*}{ Boca sana } & Dientes completos & $-0,5790$ \\
& Ausencia de placa y cálculos & 0,7794 \\
Caries dental & Descalcificación ósea & $-0,3839$ \\
\multirow{2}{*}{ Sangrado e inflamación encías } & 0,5545 \\
& Mala alimentación & $\mathrm{NA}$ \\
& Consolidado de encía & 0,09317 \\
\multirow{2}{*}{ Enseñanza cepillado } & Padres & 0,41544 \\
& Maestros & 0,43291 \\
& Odontólogos & 0,38904 \\
\multirow{2}{*}{ Creencias populares } & Radio y TV & $-0,03140$ \\
& Sola & 0,85049 \\
\hline
\end{tabular}

NA: No aplica

Tabla 3. Variables y sus criterios priorizados mediante análisis de factores para los conocimientos sobre prácticas de las gestantes en salud oral.

\begin{tabular}{llc}
\hline \multicolumn{1}{c}{ VARIABLE } & \multicolumn{1}{c}{ Criterios Priorizados } & Coeficientes de Puntajes \\
\hline \multirow{2}{*}{ Uso de elementos de higiene oral } & Uso de seda & 0,45139 \\
Momento y frecuencia de cepillado & Uso de palillos & $-0,50652$ \\
& Número de veces & 0,93199 \\
Uso de seda & Costo de la seda & 0,44154 \\
& No sabe usar la seda & 0,78918 \\
Actividades por sangrado de encías & No recuerda usarla & 0,59560 \\
Actividad por dolor dental & Uso de remedios caseros & 0,63223 \\
& Ingesta de antibióticos & 0,61899 \\
\hline
\end{tabular}

\section{Análisis bivariado de las variables priorizadas}

\section{Relacionadas con creencias}

\section{Resultados globales relacionados con creen- cias antes y después de la intervención}

Antes de la intervención, el 60\% (24) de las gestantes reportan que una boca sana es tener los dientes completos; sin embargo, después de la intervención el 40\% (16) hace esta misma afirmación. Al analizar las creencias relacionadas con la aparición de la caries dental, se observó que el 35.5\% (15) de las gestantes, antes de la intervención, respondieron que los dientes presentaban caries como efecto de la descalcificación ósea, el 85\% (24) mantuvo esta creencia después de efectuada la intervención (Tabla 4).

Previa a la aplicación del programa preventivo, el $60 \%$ (24) de las gestantes reportó que las encías sangran y se inflaman por una alimentación inadecuada. Sin embargo, el 85\% (24) hizo esta misma afirmación, después de ser intervenidas. El consolidado para la variable encía reportó antes de la intervención un promedio de -0.144 , y después de la intervención un valor de 0.613 .

Antes de la intervención, el 15\%(6) de las gestantes afirmaron que aprendieron solas a cuidar su salud oral; sin embargo, después de la intervención el 27.5\% afirmaron lo mismo. Al analizar las creencias populares de las gestantes, el $10 \%$ de 
ellas, antes de ser intervenidas reportaron que los hijos heredan la mala dentadura de los padres, después de la intervención el 2.5\% hizo la misma afirmación. Antes de la intervención, la variable consolidada para creencias mostró un promedio de 0.16 y después de la intervención aumentó a 0.61 (Tabla 4).

\section{Resultados del análisis bivariado relacio- nados con creencias antes y después de la intervención en el grupo intervenido y el grupo control}

Para la variable boca sana se observaron asociaciones estadísticamente significativas antes de la intervención para el criterio dientes completos $(p=0.022)$, ya que el $33.3 \%$ (8) de las gestantes intervenidas pensaban que una boca sana era tener los dientes completos, mientras que el $66.7 \%$ (16) de las no intervenidas afirmaron lo mismo. Para las demás variables, no se observaron asociaciones estadísticamente significativas antes de la intervención (Tabla 4).

Después de la intervención se observaron asociaciones estadísticamente significativas para el criterio dientes completos ( $p<0.001)$, ya que ninguna de las gestantes intervenidas reportó que una boca sana era tener los dientes completos, mientras que el $100 \%$ (16) de las gestantes no intervenidas, afirmaron lo mismo. (Tabla 4)

$\mathrm{Al}$ analizar las creencias relacionadas con caries dental se encontraron asociaciones estadísticamente significativas para la variable descalcificación ósea $(\mathrm{p}<0.001)$ después de la intervención. El 79.2\% (19) de las gestantes intervenidas afirmaron que la caries dental era causada por la descalcificación ósea, mientras que el $28.8 \%$ (5) del grupo no intervenido, opinaron lo mismo. Sin embargo, el 58.8\% (20) del grupo de las gestantes intervenidas pensaban que la caries dental era producida por una mala alimentación, y sólo el $41.1 \%$ (14) del grupo no intervenido mantenía esta creencia.

Después de la intervención, para el consolidado de la variable encía se observaron asociaciones estadísticamente significativas $(p<0.001)$, con promedios de 0.613 en el grupo intervenido, y de -0.613 para el grupo no intervenido. De la misma manera, se observaron cambios para la variable consolidado de creencias populares con un promedio de 0.28 en el grupo intervenido y de -0.28 en el grupo no intervenido y para la variable consolidada de creencias definitivas se observaron promedios de 0.75 para el grupo intervenido, y de -0.75 para el grupo no intervenido (Tabla 4).

\section{Relacionado con prácticas}

\section{Resultados globales relacionados con prác- ticas antes y después de la intervención}

$\mathrm{Al}$ analizar las prácticas relacionadas con los elementos de higiene oral, el 65\% (26) de las gestantes antes de ser intervenidas, afirmaron que entre sus elementos de higiene oral utilizaban la seda dental para la limpieza de sus dientes; sin embargo, el 90\% (36) de las gestantes afirmaron lo mismo después de ser intervenidas. El 27.5\% (11) de las gestantes dijeron que utilizaban palillos como elementos de higiene oral. Sin embargo, después de la intervención el 15\% (6) de las gestantes mantuvieron esta práctica (Tabla 5).

Al evaluar el momento y la frecuencia del cepillado en las gestantes, se encontró que antes de la intervención el promedio de veces de cepillado al día era de 3.3, y después de la intervención aumentó a 4.1 (Tabla 5).

Con relación al uso de la seda dental, antes de la intervención el 37.5\% (15) de las gestantes manifestaron que no la utilizan porque no recordaban hacerlo. Después de la intervención, el 25\% (10) de ellas afirmaron lo mismo. Al analizar las actividades realizadas para el sangrado de las encías se encontró que el $12.5 \%$ (5) de las gestantes antes de ser intervenidas, afirmaron que emplean remedios caseros y el 5\% (2) manifestaron lo mismo después de la intervención (Tabla 5).

\section{Resultados del análisis bivariado relacio- nados con prácticas antes y después de la intervención en el grupo intervenido y el grupo control}

No se observaron asociaciones estadísticamente significativas entre el grupo intervenido y el no intervenido, antes de la intervención para las variables de prácticas. Al analizar las prácticas relacionadas con el uso de elementos para la limpieza de los dientes, se observaron asociaciones estadísticamente significativas después de la intervención, para la variable uso de palillos $\left(0.020^{* *}\right)$, ya que ninguna de las gestantes del grupo intervenido utilizaba los palillos como elemento de higiene oral. Después de la intervención, el 100\% (6) del grupo no intervenido realizaba dicha práctica.

Después de la intervención el grupo de las gestantes intervenidas obtuvo un promedio de número de veces de cepillado al día de 5.1, mientras 
que en el grupo de las no intervenidas fue de 3.2. En relación con el uso de la seda dental, se observó que después de la intervención, el 10\% (1) del grupo intervenido manifestó que no utiliza la seda porque se le olvida, mientras que el $90 \%$ (9) del grupo no intervenido afirmó lo mismo
Para la variable consolidado de prácticas, se mostraron asociaciones estadísticamente significativas ya que el promedio del grupo intervenido mostró valores de 0.536 , mientras que el grupo no intervenido obtuvo valores de -0.536 (Tabla 5).

Tabla 4.Variables priorizadas definitivas para creencias en salud oral analizadas de forma global antes y después del programa preventivo.

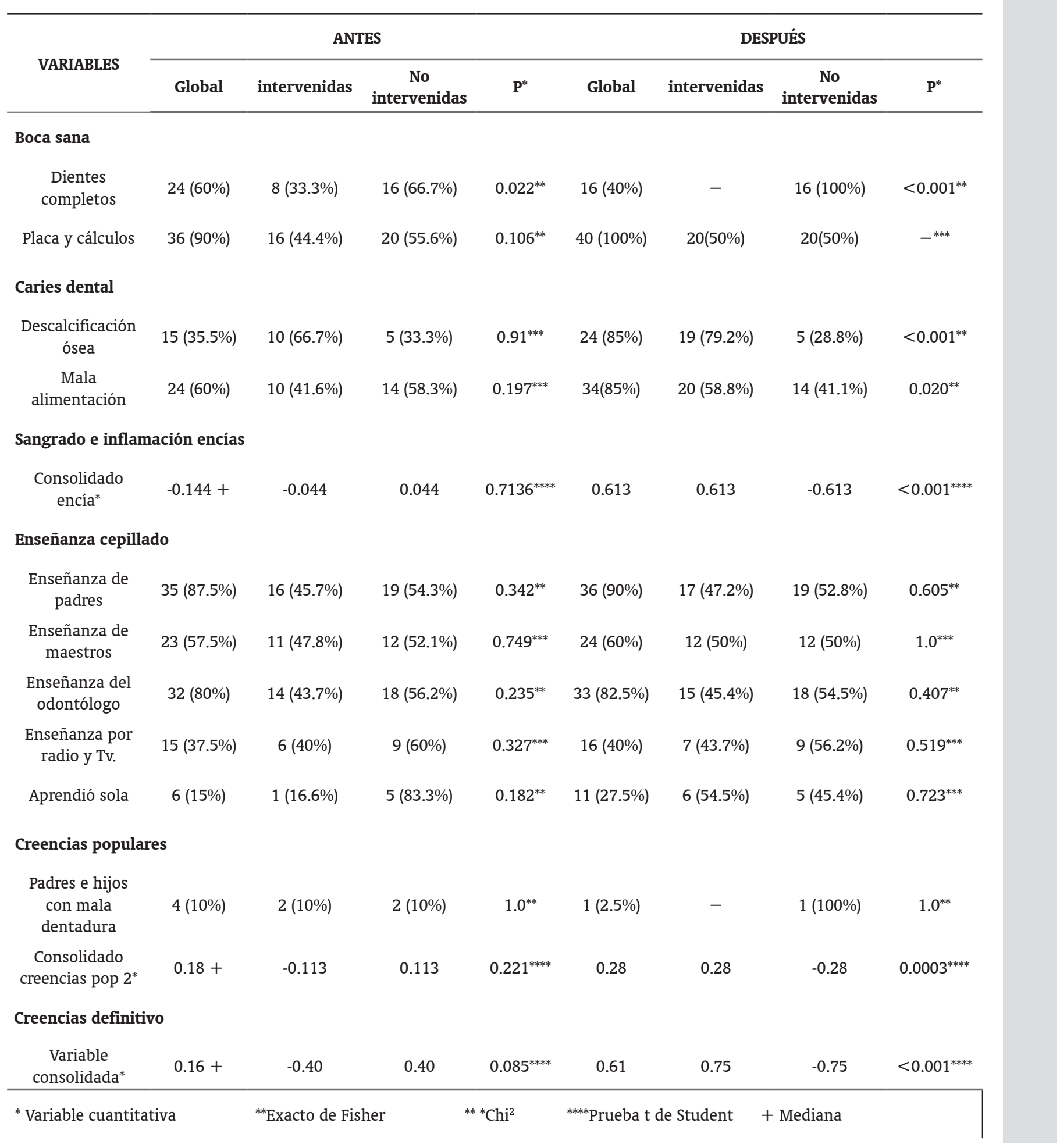


Tabla 5. Variables priorizadas, generadas y definitivas para prácticas en salud oral analizadas de forma global antes y después del programa preventivo.

\begin{tabular}{|c|c|c|c|c|c|c|c|c|}
\hline \multirow{2}{*}{ VARIABLES } & \multicolumn{4}{|c|}{ ANTES } & \multicolumn{4}{|c|}{ DESPUÉS } \\
\hline & Global & Intervenidas & $\begin{array}{c}\text { No } \\
\text { intervenidas }\end{array}$ & $\mathbf{P}^{*}$ & Global & intervenidas & $\begin{array}{c}\text { No } \\
\text { intervenidas }\end{array}$ & $\mathbf{P}^{*}$ \\
\hline \multicolumn{9}{|c|}{ Uso de elementos de higiene oral } \\
\hline Uso de seda & $26(65 \%)$ & $11(42.3 \%)$ & $15(57.7 \%)$ & $0.185^{* * *}$ & $36(90 \%)$ & $20(55.5 \%)$ & $16(44.4 \%)$ & $0.106^{* *}$ \\
\hline Uso de palillos & $11(27.5 \%)$ & $5(45.5 \%)$ & $6(54.5 \%)$ & $0.723^{* * *}$ & $6(15 \%)$ & - & $6(100 \%)$ & $0.020^{* *}$ \\
\hline \multicolumn{9}{|c|}{ Momento y frecuencia del cepillado } \\
\hline $\begin{array}{l}\text { No. veces } \\
\text { cepillado * }\end{array}$ & $3.3+$ & 3.3 & 3.25 & $0.895^{* *}$ & 4.1 & 5.1 & 3.2 & $<0.001^{* *}$ \\
\hline \multicolumn{9}{|c|}{ Uso de la seda dental } \\
\hline $\begin{array}{l}\text { Costo de la } \\
\text { seda }\end{array}$ & $5(12.5 \%)$ & $1(20 \%)$ & $4(80 \%)$ & $0.342^{* *}$ & $5(12.5 \%)$ & $1(20 \%)$ & $4(80 \%)$ & $0.342^{* *}$ \\
\hline $\begin{array}{c}\text { No sabe usar la } \\
\text { seda }\end{array}$ & $6(15 \%)$ & $2(33.3 \%)$ & $4(66.6 \%)$ & $0.661^{* * *}$ & $5(12.5 \%)$ & $1(20 \%)$ & $4(80 \%)$ & $0.342^{* *}$ \\
\hline $\begin{array}{l}\text { No se acuerda } \\
\text { de usar la seda }\end{array}$ & $15(37.5 \%)$ & $6(40 \%)$ & $9(60 \%)$ & $0.327^{* *}$ & $10(25 \%)$ & $1(10 \%)$ & $9(90 \%)$ & $0.008^{* *}$ \\
\hline \multicolumn{9}{|c|}{ Actividades por sangrado de encías } \\
\hline $\begin{array}{l}\text { Uso de } \\
\text { remedios } \\
\text { caseros }\end{array}$ & $5(12.5 \%)$ & $2(40 \%)$ & $3(60 \%)$ & $1.0^{* *}$ & $2(5 \%)$ & - & $2(100 \%)$ & $0.487^{* *}$ \\
\hline \multicolumn{9}{|c|}{ Actividades por dolor dental } \\
\hline $\begin{array}{c}\text { Uso de } \\
\text { antibióticos }\end{array}$ & $10(25 \%)$ & $4(40 \%)$ & $6(60 \%)$ & $0.716^{* *}$ & $7(17.5 \%)$ & $1(14.3 \%)$ & $6(85.7 \%)$ & $0.091^{* *}$ \\
\hline \multicolumn{9}{|c|}{ Prácticas definitiva } \\
\hline $\begin{array}{c}\text { Variable } \\
\text { consolidada* }\end{array}$ & $0.33+$ & 0.11 & -0.11 & $0.3873^{* * * *}$ & 0.37 & 0.536 & -0.536 & $0.0001^{* * * *}$ \\
\hline
\end{tabular}

\section{DISCUSIÓN}

Es de vital importancia que la gestante comprenda que la práctica odontológica no representa una amenaza para su salud. Esta situación se logra, si el profesional posee un conocimiento adecuado y le proporciona una información pertinente que le brinde la suficiente confianza y seguridad.

En este estudio, la mayoría de las gestantes eran casadas (42.5\%), habían terminado su bachillerato, trabajaban fuera de la casa (52.5\%), eran madres primerizas $(72.5 \%)$, la mitad de ellas habían programado su embarazo y eran mujeres jóvenes de 25.33 años promedio. ${ }^{14}$ Estos datos están próximos a los obtenidos por Saldarriaga y colaboradores, Garbero y colaboradores, y García en los que la edad media encontrada era de $26 \pm 6.38$ años. Estos factores han sido reportados en la literatura como protectores para la aparición de caries dental en las madres y sus hijos. ${ }^{2,15,22,23}$

En la gestación, las madres están sujetas a diferentes cambios tanto en su cuerpo como en sus emociones. Por tal razón, la atención y el cuidado se torna un poco más escrupuloso y dedicado. Sin embargo, no quiere decir que ellas sean personas incapacitadas para realizar las actividades de su vida cotidiana.

La asistencia a la consulta odontológica por parte de las mujeres se ve seriamente afectada cuando 
se encuentra en este periodo de la vida. Saldarriaga y colaboradores, en el 2003, reportaron que el trimestre en el que más consultaron las madres al odontólogo fue el segundo (47.5\%), seguido por el tercero (39.1\%). Esta situación es preocupante ya que en el primer trimestre se hace menos frecuente la consulta, siendo esta etapa la más adecuada para implementar acciones de promoción y prevención en salud oral y establecer rutinas saludables. ${ }^{22,24}$

Identificar y analizar las prácticas y las creencias de las gestantes relacionadas con la higiene oral va a permitir conocer lo que piensan y sienten, así como establecer los motivos por los cuales no acuden a la consulta odontológica y las acciones que reemplazan. ${ }^{7,24}$.

Este grupo poblacional no considera la presencia de caries, gingivitis, movilidad dental e incluso tener la cara inflamada, como un estado de enfermedad; lo que visto desde la perspectiva de la salud, constituye un problema significativo por las repercusiones sobre la salud general de la madre y del bebé al no ser diagnósticadas y tratadas adecuada y oportunamente.

Antes de la intervención, las madres mostraron diferencias estadísticamente significativas con relación a la creencia que una boca sana es tener los dientes completos ( $\mathrm{p}=0.022)$; el $(33.3 \%)$ del grupo intervenido y $(66.7 \%)$ del grupo no intervenido tenían esta creencia. En las demás variables no se observaron diferencias estadísticamente significativas.

De lo anterior, se puede deducir que para las gestantes la estética dental es significado de salud sin importar si hay presencia de caries dental o de enfermedad periodontal. Nieva y colaboradores (1999) resaltaron cómo las personas encuestadas respondieron que tener una boca saludable es "tener unos dientes parejos, completos, sin huecos, con una buena sonrisa". ${ }^{11 .}$

Se han implementado algunos programas preventivos en las embarazadas para evaluar si podrían llevarlas a mejorar su actitud con relación al cuidado de su salud dental y al mismo tiempo, mejorar la salud general de madre e hijo. ${ }^{14,25}$

Después de la aplicación del programa preventivo, ninguna gestante del grupo intervenido afirmó que tener una dentadura completa es indicador de una boca sana, mientras que el $100 \%$ de las gestantes del grupo no intervenido afirmaron lo anterior. $\mathrm{Al}$ analizar las creencias en cuanto a la caries dental, se encontró que el $79.2 \%$ de las madres del grupo intervenido pensaban que la caries se pro- ducida por la descalcificación ósea, mientras que el $28.8 \%$ del grupo no intervenido afirmaron lo mismo. Se ha reportado que el proceso de la caries dental se produce por descalcificación del esmalte dentario debido a factores locales, más no por la descalcificación de los huesos. 8,26-29

Entre las causas que atribuyen las gestantes a la aparición de caries dental está la mala alimentación donde se observaron asociaciones estadísticamente significativas ( $\mathrm{p}=0.020)$; después de la intervención, el 58.8\% de las gestantes del grupo intervenido tenía esta creencia mientras que el $41.1 \%$ del grupo no intervenido la mantuvo. Saldarriaga y colaboradores reportaron que el $7.6 \%$ de las gestantes encuestadas afirmaron que la mala alimentación es un factor que predispone para la aparición de la caries dental. ${ }^{22}$

Las alteraciones gingivoperiodontales son reacciones conectivo-vasculares de la encía, por acción de un agente irritante, ya sea placa bacteriana o cálculos dentales. En el embarazo se producen cambios hormonales que elevan la progesterona y los estrógenos; se ha demostrado que estas alteraciones hormonales tienen influencia sobre el sistema inmune y contribuyen significativamente en la etiología y patogénesis de la gingivitis del embarazo. . $^{1,2,3,11,30 \text {. }}$

Para la variable causas del sangrado de las encías, se generó un nueva variable (encía) la cual consolidó las demás variables (mal cepillado o no cepillado, cepillado fuerte, problemas en el organismo, mala alimentación), en la que se observaron diferencias estadísticamente significativas $(p<0.001)$. Después de la intervención se reportaron promedios de 0.613 y de -0.613 para el grupo intervenido y no intervenido, respectivamente.

Para las variables enseñanza de cepillado y creencias populares, no se observaron asociaciones estadísticamente significativas antes y después de la intervención entre los dos grupos de gestantes; sin embargo, los resultados globales indican que antes de la intervención el $15 \%$ de las gestantes afirmaron que aprendieron solas a cuidar su salud oral y después de la intervención, el 27.5\% manifestaron lo mismo. De igual manera, antes de la intervención el $10 \%$ de las gestantes afirmaron que cuando los padres tenían mala dentadura los hijos la heredaban, y después de la intervención sólo el $2.5 \%$ afirmó lo mismo. Saldarriaga y colaboradores reportaron que el $87 \%$ y $76.2 \%$ de las gestantes de EPS y ARS, respectivamente, opinaban que no es cierto que los hijos tenían mala dentadura porque la heredan de sus padres, lo cual es muy similar en el presente estudio, ya que un bajo porcentaje de 
las madres respondieron afirmativamente a esta creencia después de la intervención. ${ }^{11,22}$

Las prácticas sociales tanto preventivas como de tratamiento, referidas por la población del sector, derivan sus orígenes en la tradición familiar por las creencias de madres, abuelas, personas mayores y vecinos. Igualmente, están estrechamente relacionadas con la percepción que se tiene de las causas de la enfermedad. ${ }^{11}$

El uso de elementos de higiene oral, es un factor importante para la adecuada salud oral de las gestantes, y en general de toda la población. Aunque la literatura no reporta resultados sobre el uso de los palillos como elemento de higiene oral, para esta variable se observaron asociaciones estadísticamente significativas entre el grupo intervenido y el grupo control, después de la intervención $(p=0.020)$, mientras que para el uso de la seda, no se observaron asociaciones. Sin embargo, los resultados globales nos muestran que antes de la intervención, el 65\% de las gestantes hacían uso de la seda, después de la intervención el $90 \%$ de las gestantes seguían realizando esta práctica, lo que puede sugerir que estas diferencias fueron producto de la aplicación del programa preventivo.

García reportó en un estudio constituido por 97 gestantes adolescentes y 153 gestantes adultas que asistieron al control prenatal de las IPS Las Américas, San Luis y San Francisco de Asís en la cuidad de Sincelejo que el $79.08 \%$ de las gestantes adultas realizaban con más frecuencia el cepillado de dientes, en contraste con el $73.20 \%$ en las gestantes adolescentes. ${ }^{23}$

Para la variable número de veces de cepillado al día se encontraron asociaciones estadísticamente significativas $(\mathrm{p}<0.001)$ con promedios de 5.1 y 3.3 para el grupo intervenido y el grupo control respectivamente, después de la intervención. Con relación al no uso de la seda dental por olvido, se observaron asociaciones estadísticamente significativas $(p=0.008)$ entre los dos grupos después de la intervención, el intervenido con el $10 \%$ y el control con el $90 \%$. Esto conduce a pensar que las gestantes del grupo intervenido tuvieron un comportamiento receptivo al programa preventivo.

La utilización de remedios caseros como plantas medicinales, sal, bicarbonato, ceniza, entre otros, son prácticas sociales muy comunes e importantes en el mantenimiento de la salud oral porque resuelve, a bajos costos y en un alto porcentaje, sus problemas inmediatos. ${ }^{11}$

Frente al sangrado e inflamación de la encías llamado popularmente como "piorrea", la comunidad manifiesta el uso del romero, una planta medicinal que se utiliza como colutorio. Esta práctica es muy similar a la de las gestantes de nuestro estudio, ya que, aunque no se observaron asociaciones estadísticamente significativas, los resultados globales reportaron que antes de la intervención el 12.5\% utilizaban remedios caseros para evitar el sangrado de las encías, mientras que después de la intervención, el $5 \%$ hacían lo mismo.

Finalmente, para la variable consolidada de prácticas definitivas los promedios obtenidos después de la intervención para el grupo intervenido fue de 0.536 y para el no intervenido de -0.536 , se observó que después de la aplicación del programa preventivo hubo asociaciones estadísticamente significativas entre los dos grupos $(\mathrm{p}=0.0001)$.

El grupo gestacional es reconocido por la legislación colombiana, como vulnerable y primordial en la atención; ${ }^{4}$ no obstante, los profesionales de la salud no son conscientes del gran impacto que causa la atención odontológica en la madres sobre sus creencias y prácticas en salud oral. Por lo tanto, es necesario que los odontólogos y demás profesionales de la salud incentiven la asistencia a la cita odontológica durante el primer trimestre, siendo esta la etapa más adecuada, ya que al iniciar el embarazo se producen cambios hormonales que conllevan a la alteración del periodonto..$^{14,22-28}$

Hay un gran desconocimiento sobre los dogmas y las prácticas adecuadas en salud oral por parte del grupo gestante. Los resultados de este estudio revelaron que las gestantes antes de ser intervenidas tenían conceptos muy diferentes a los que presentaron después de la intervención. Los programas preventivos en salud oral, se hacen cada vez más necesarios, para que de una u otra manera las gestantes comprendan la importancia de sus dientes y tejidos adyacentes, sobre todo, en esta etapa de la vida. ${ }^{2,11,12,14,22}$

La aceptación de estilos de vida saludables y de hábitos de autocuidado deberían incluirse en periodos tempranos de la vida, con el propósito de aumentar la probabilidad de que se mantengan; por tal razón, iniciar programas de educación en salud orientados a fortalecer comportamientos saludables deberían iniciarse en el hogar y en las instituciones de educación primaria. ${ }^{4}$ Tales estudios son de gran valor, ya que permiten evidenciar la realidad de nuestra población, sus pensamientos, sentimientos y actividades que realizan diariamente. Es importante que estas investigaciones se lleven a cabo, puesto que conocer lo que piensan y hacen los pacientes es de vital importancia al momento de la consulta. 
Las fortalezas captadas de este trabajo se basan en la información que aporta una exploración detallada de información secundaria y que demuestra la importancia de retomar bases de datos que existen con el propósito de clarificar aspectos adicionales que se pueden considerar en un proceso investigativo que ya se realizó. Es importante destacar además, que con el análisis detallado de los aspectos involucrados en este proceso analítico se pueden clarificar las temáticas que se deben reforzar en la implementación de un programa preventivo para lograr mejores resultados, pues es de gran ayuda para conocer hábitos adecuados e inadecuados de las mujeres gestantes. De igual manera, ayuda a que conozcan los verdaderos y adecuados hábitos en salud oral.

Las potenciales limitaciones también se relacionan con la utilización de fuentes secundarias y por el hecho que las responsables de este proceso investigativo derivado del ECC realizado previamente, encontraron dificultades en la interpretación de algunas preguntas del instrumento y, por ende, del esclarecimiento de algunos resultados relacionados con la exploración desarrollada.

Los resultados del presente trabajo motivan a continuar la implementación de programas preventivos en los diferentes lugares donde se realiza la práctica odontológica, ayudan a prevenir las enfermedades de salud oral, y mejoran la calidad de vida de las mujeres gestantes. De igual manera, surge el interés de aplicar este programa preventivo en una empresa promotora de salud que incluya a gestantes del régimen subsidiado de Bucaramanga con el fin de evidenciar, si los factores económicos influyen sobre las prácticas y creencias de las madres.

\section{CONCLUSIONES}

La priorización de las variables que identifican las prácticas y creencias relacionadas con la salud oral en las gestantes es un proceso que permitió la realización de un estudio más reducido y consistente con el fin de obtener unos resultados más concretos.

Las características sociodemográficas de las gestantes indican que hay pocos factores de riesgo para su etapa de gestación. Se podría concluir que estas madres llevan un embarazo más sereno, a diferencia de otras, que no tienen estas mismas características o condiciones, ya que la mayoría de ellas habían terminado su bachillerato, la mitad trabaja fuera de la casa y habían programado su embarazo, más de la mitad eran madres primerizas y eran mujeres jóvenes pero no adolescentes.
Las prácticas y creencias que las gestantes manifestaron antes de la intervención son factores de riesgo agravantes para la aparición de la caries dental y la enfermedad periodontal. La adecuada aplicación de un programa preventivo ayuda a mejorar las prácticas y creencias de salud oral en las mujeres gestantes.

\section{BIBLIOGRAFÍA}

1. Rodríguez HE, López M. El embarazo. Su relación con la salud bucal. Rev Cubana Estomatol [en línea] 2003; 40 [fecha de acceso: septiembre de 2008]. URL disponible en: http:// bvs.sld.cu/revistas/est/vol40_2_03/est09203.htm

2. Garbero I, Delgado AM, Benito IL. Salud oral en embarazadas: Conocimientos y actitudes. Acta Odontol Venez [en línea] 2005: 43 (2) [fecha de acceso: agosto de 2008]. URL disponible en: http://www.actaodontologica. com/ediciones/2005/2/salud_oral_embarazadas.asp

3. Rodríguez MC. Nivel de conocimiento sobre prevención en salud bucal en gestantes del Hospital Nacional Daniel A. Carrión en el año de 2002. Trabajo de Grado [en línea]. URL disponible en: http://sisbib.unmsm.edu.pe/BibVirtual/tesis/Salud/Rodriguez_V_M/indice.htm

4. República de Colombia. Resolución 00412 de 2000. Norma Técnica para la Detección Temprana de las Alteraciones del Embarazo.

5. Valadez I, Alfaro N, Pozos E. Cuidados alternativos en la atención del embarazo en Jalisco, Méxido. Cad Saúde Pública [en línea] 2003; 19: 1313 - 1321. URL disponible en: http://www.scielosp.org/pdf/csp/v19n5/17804.pdf

6. Domínguez E, flores JI, Hernández I, Mireles AJ, Perera A, Tolosa JS, Graf A. Perfil de las pacientes embarazadas que acuden a las clínicas odontológicas periféricas de las FES ISTALACA [en línea]. URL disponible en: http:// odontologia.iztacala.unam.mx/instrum_y_lab1/otros/ ColoquioXVI/contenido/indice_cartel_archivos/TRAB\%20 COMPL\%20COLOQ\%20CARTEL/HTML/1302.htm

7. Díaz RRM, Carrillo CM, Terrazas RJF, Canales MCE. Actitudes que influyen en la demanda de servicios odontológicos durante la gestación. Revista ADM 2001; 58: 68 - 73.

8. Herrera CL, Pantoja P, de La Maza T, Sanhueza A, Salazar LA. Diagnóstico microbiológico y molecular de bacterias cariogénicas en mujeres embarazadas de la Región de La Araucanía, Chile. Rev Chil Infect 2007; 24: 270 - 275.

9. Arrufat Nebota FJ. Diagnóstico de los trastornos mentales del embarazo y el puerperio Viernes 28 Abril 2000. Volumen 58 - Número 1342 p. 57 - 60.

10. Olivar Bonilla A. Cambios biológicos: psicológicos y sociales durante el embarazo. Revista de la Federación Odontológica Colombiana. 201: 37 - 51.

11. Nieve B, Jácome S, Cendales L. Representaciones sociales del proceso salud enfermedad oral en poblaciones urbano - marginales y su relación con los discursos y las prácticas institucionales. Revista de la Federación Odontológica Colombiana 1999; 57: 9 - 56.

12. Ferreira AR, Benfatti SV, Bausells J. Rev Ibero-Am Odontopeditr Odontobebe 2004; 7: 66 - 77.

13. Rojas J. Características psicosociales y de salud de gestantes que desean o no su embarazo. Revista Comportamiento [en línea]. 2005: 7: 35 - 44. URL disponible en: http://www.comportamiento.dsm.usb.ve/revista/vol_7_2/ rojas2005.pdf 
14. Riveros $\mathrm{CM}$, Rodríguez MJ. Programa preventivo en salud oral para controlar factores de riesgo asociados a caries dental y enfermedad periodontal en mujeres gestantes. Ustasalud 2007; 6: 17 - 28.

15. Republica de Colombia. Ministerio de Salud. Tercer Estudio Nacional de Salud Bucal- ENSAB III. Documentos Técnicos. Tomo VII. Bogotá, 1999.

16. Microsoft office Excel 2003. Microsoft Corporation.

17. CDS-OMS. Epi-info 6, Versión 6.04. Atlanta, (Nov 1996).

18. Stata Corp. Stata Statistical Software. Release 8.0. Collage station. TX: Stata corporation 2003.

19. Pagano M, Gauvreau K. Principles of Bioestatistics. Belmont: Duxbury Press. (1995) p. 34-44, 257-263.

20. Norman GR, StreinerDL. Bioestadística. Madrid: Mosby/ Doyma Libros, 1996. p. 129-142.

21. República de Colombia. Ministerio de Salud. Resolución 008430 de 1993. URL disponible en: www.ins.gov.co/pdf/ admon/juridica/ei/ei 1993 res ms 8430.pdf

22. Saldarriaga OJ, Sánchez M, Avendaño L. Conocimientos y prácticas en salud bucal de las gestantes vinculadas al programa de control prenatal Medellín 2003. Revista CES Odontología 2004; 17: 9 - 23.

23. García LM. Comparación de las prácticas de cuidado en dos grupos de gestantes en la ciudad de Sincelejo. AV Enferm 2008; XXVI: 22 - 35.

24. Moynihan P. The role of diet and nutrition in the etiology and prevention of oral diseases. Bulletin of the World Health Organization 2005; 83: $694-699$.

25. Pérez JA, Duque J, Hidalgo I. Asociación del Estreptococos mutans y lactobacilos con la caries dental en niños. Matanzas Cuba 2004 -2005. Facultad de Ciencias Médicas de Matanzas. CUBA

26. Microbiología de la caries dental. Acta Odontol Venez [en línea] 2003; 41: 293 - 294 [fecha de acceso: 12 julio de 2008]. URL disponible en: http://www.scielo.org.ve/scielo.php?pid $=$ S0001-63652003000300018\&script $=$ sci_arttext

27. Ekstrand KR, Rickets DNJ, Kidd EAM. Occlusal caries: Pathology, diagnosis and logical management. Dent Update 2001; $28: 380-387$.

28. Almarales C, Llerandi Y. Conocimientos sobre salud bucal y demanda de servicios estomatológicos en relación con la enfermedad periodontal en embarazadas. Rev Cubana Estomatol [en línea] 2008; [fecha de acceso: mayo de 2008] 45 (2). URL disponible en:// scielo.sld.cu/scielo.php?script $=$ sci arttext\&pid $=$ S0034$75072008000200003 \& \operatorname{lng}=\mathrm{es} \& \mathrm{nrm}=$ iso-

29. Taboada V, Mendoza D, Hernández I, Martínez A. Prevalencia de caries dental en un grupo de pacientes de la tercera edad. México. Revista ADM 2000; 57: 188 - 192.

30. Suresh L, Radfar L. Pregnancy and lactation. Oral Surg Oral Med Oral Pathol Oral Radiol Endod 2004; 97: 672 -682 .

31. Tascon. J.E, Aranzazu.L, Velasco. T, Trujillo. K, Paz. M. Primer molar permanente historia de caries en un grupo de niños entre los 5 y 11 años frente a los conocimientos, actitudes y prácticas de sus madres. Colombia Medica 2005: 36: 41 - 46.
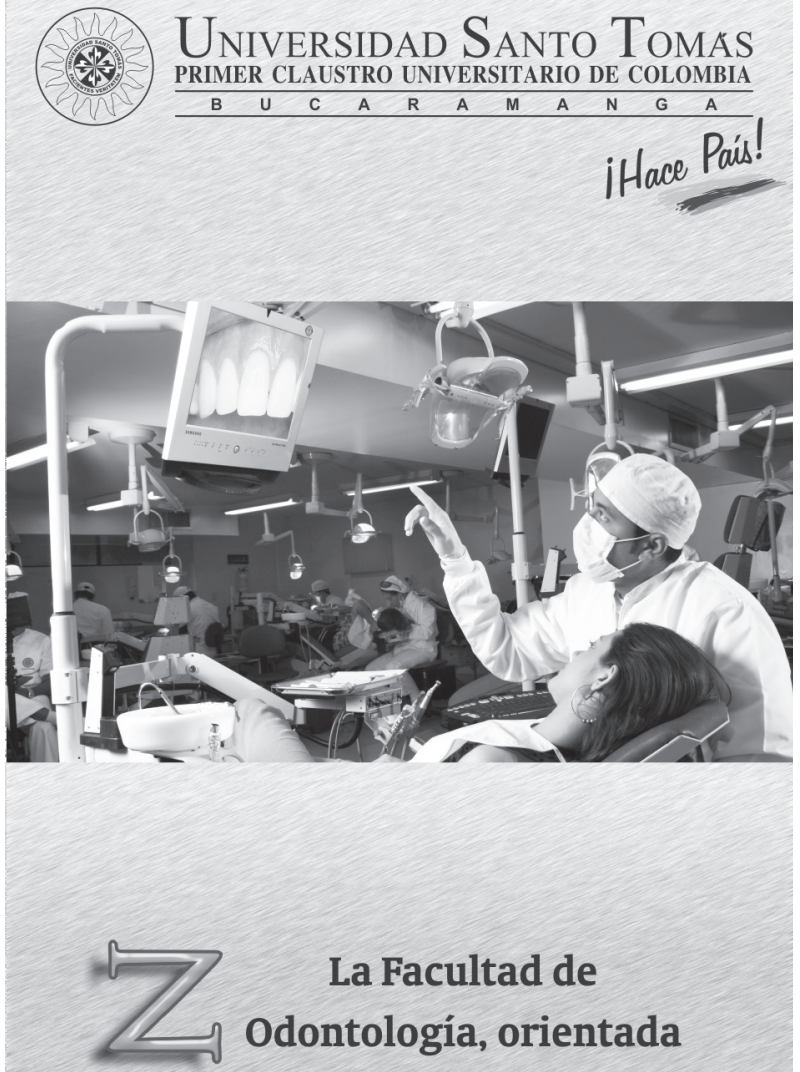

La Facultad de

Odontología, orientada por el pensamiento de Santo Tomás, pretende

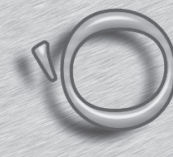
formar Odontólogos integrales $\mathrm{y} / 0$ especialistas a través de parámetros humanísticos, éticos, científicos, biotecnológicos, investigativos y

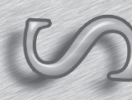
sociales, como recurso humano capaz de

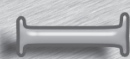
intervenir con éxito en el proceso dinámico de la salud y la enfermedad, en el individuo, la familia y la comunidad. 